William W. Adams

Nagoya Math. J.

Vol. 42 (1971), 79-87

\title{
SIMULTANEOUS ASYMPTOTIG DIOPHANTINE APPROXIMATIONS TO A BASIS OF A REAL NUMBER FIELD
}

\author{
WILLIAM W. ADAMS
}

\section{Introduction}

The purpose of this paper is to prove the following result.

Theorem 1. Let $K$ be a real algebraic number field of degree $m=n+1$. Let $1, \beta_{1}, \cdots, \beta_{n}$ be a basis of $K$. For a given constant $C>0$ set $\lambda_{B}=\lambda_{B}\left(\beta_{1}, \cdots\right.$, $\left.\beta_{n}, C\right)$ equal to the number of solutions in integers $q, p_{1}, \cdots, p_{n}$ of the inequalities

$$
\begin{array}{lll}
* & 0<q \beta_{i}-p_{i}<C / q^{\frac{1}{n}} & (1 \leq i \leq n) \\
* & 1 \leq q \leq B . &
\end{array}
$$

Then $\lambda_{B}=0(1)$ or there is a $C^{\prime}>0$ such that $\lambda_{B} \sim C^{\prime} \log B(B \rightarrow \infty)$.

There is a dual theorem.

Theorem 2. With $\beta_{1}, \cdots, \beta_{n}, C$ as in Theorem 1 set $\Lambda_{B}$ equal to the number of solutions in integers $q_{1}, \cdots, q_{n}, p$ of the inequalities

$$
\begin{gathered}
0<q_{1} \beta_{1}+\cdots+q_{n} \beta_{n}-p<C / q^{n} \\
1 \leq q_{1}, \cdots, q_{n} \leq B
\end{gathered}
$$

where $q=\max \left(q_{1}, \cdots, q_{n}\right)$. Then either $\Lambda_{B}=0(1)$ or there is a $C^{\prime \prime}>0$ such that $\Lambda_{B} \sim C^{\prime \prime} \log B(B \rightarrow \infty)$.

These results generalize the results of $[2,3]$. However the work of $[2,3]$ had the advantage that the constant $C^{\prime}$ was more precisely defined. For related work see $[1,4,5]$.

The author would like to thank W.M. Schmidt and S. Kuroda for not believing that the results of [2] could not be generalized to higher dimensions.

Received April 28, 1970. 
The proof of the present result closely parallels the proof in [2]. Hence this paper will sometimes be sketchy.

\section{The reduction to counting units}

Denote by $\boldsymbol{Z}, \boldsymbol{Q}, \boldsymbol{R}, \boldsymbol{C}$ the integers, rationals, reals and complexes. Let $\tau_{0}=$ identity, $\tau_{1}, \cdots, \tau_{n}$ denote the distinct embeddings of $K$ into $\boldsymbol{C}$. Assume $\tau_{0}, \cdots, \tau_{r}$ are the real embeddings. For $\alpha \in K$, set $\tau_{i} \alpha=\alpha^{(i)}$ and assume as usual $n=r+2 s$ and $\alpha^{(r+i)}=\bar{\alpha}^{(r+s+i)}(1 \leq i \leq s)$.

Lemma 1. The dual basis $\alpha_{0}, \alpha_{1}, \cdots, \alpha_{n}$ of $K$ (with respect to the trace) has the following properties

$$
\begin{aligned}
& \alpha_{0}^{(i)}+\alpha_{1}^{(i)} \beta_{1}+\cdots+\alpha_{n}^{(i)} \beta_{n}=0(1 \leq i \leq n) \\
& \alpha_{0}+\alpha_{1} \beta_{1}+\cdots+\alpha_{n} \beta_{n}=1 \\
& A=\left(\alpha_{j}^{(i)}\right) \quad(1 \leq i, j \leq n) \text { is nonsingular. }
\end{aligned}
$$

For the proof see $[2,5]$.

Let $M$ be the free $\boldsymbol{Z}$-module generated by $\alpha_{0}, \cdots, \alpha_{n}$. Let $\mathcal{O}$ be the associated order. Let $U$ be the group of units of $\mathcal{O}$. Then by the Dirichlet Unit Theorem there are units $\zeta_{1}, \cdots, \zeta_{r+s}>1$ in $U$ such that $U=\left\{ \pm \zeta_{1}^{\nu_{1}} \cdots \zeta_{r+s}^{\nu_{r+s}}\right\}$. For $\nu=\left(\nu_{1}, \cdots, \nu_{r+s}\right) \in Z^{r+s}$ set $\zeta^{\nu}=\zeta_{1}^{\nu_{1}} \cdots \zeta_{r+s}^{\nu_{r+s}}$ so $U=\left\{ \pm \zeta^{\nu}\right\} \quad\left(\nu \in \boldsymbol{Z}^{r+s}\right)$.

For $\xi_{1}, \xi_{2} \in M$ write $\xi_{1} \sim \xi_{2}$ if there is a $\zeta \in U$ such that $\xi_{1}=\zeta \xi_{2}$ (this is an equivalence relation). If $\Omega \subseteq M$ is an equivalence class, then for all $\xi \in M$, $|\boldsymbol{N} \boldsymbol{\xi}|$ is the same and we denote this by $\boldsymbol{N} \Omega(\boldsymbol{N}$ denotes the norm of $K / \boldsymbol{Q})$.

Now for $\zeta \in M$ we have unique $q, p_{1}, \cdots, p_{n} \in Z$ such that $\xi=q \alpha_{0}+p_{1} \alpha_{1}$ $+\cdots+p_{n} \alpha_{n}$. In this way we view $\xi \in M$ as being in 1-1 correspondence with possible solutions to ${ }^{*}$ and ${ }^{* *}$. Moreover by (1) we see

$$
-\xi^{(i)}=\alpha_{1}^{(i)}\left(q \beta_{1}-p_{1}\right)+\cdots+\alpha_{n}^{(i)}\left(q \beta_{n}-p_{n}\right) \quad(1 \leq i \leq n) .
$$

Lemma 2. There are only finitely many classes $\Omega \subseteq M$ which yield solutions to *.

Proof. Combine * and (4) with the known fact that there are only finitely many $\Omega$ with $N \Omega$ below a given value.

It suffices to show the following: if for a fixed class $\Omega \subseteq M$ there are infinitely many solutions to ${ }^{*}$ then there is a $C_{1}>0$ such that the number of $\xi \in \Omega$ satisfying $*$ and $* *$ is asymptotic to $C_{1} \log B$.

We now need some notation. Set $\Omega=\left\{ \pm \zeta^{\nu} \xi_{0}\right\}\left(\nu \in \boldsymbol{Z}^{r+s}\right)$ for any $\xi_{0}>0$ in $\Omega$. Let $\Omega^{+}\left(\Omega^{-}\right)$denote the positive (negative) elements for $\Omega$. Let 
$\xi_{\nu}=\zeta^{\natural} \xi_{0}\left(\nu \in \boldsymbol{Z}^{r+s}\right)$ denote a typical element of $\Omega^{+}$. Set

$$
\xi_{\nu}=q_{\nu} \alpha_{0}+p_{1 \nu} \alpha_{1}+\cdots+p_{n \nu} \alpha_{n} .
$$

Write

$$
\gamma_{i \nu}=q_{\nu} \beta_{i}-p_{i \nu} \quad(1 \leq i \leq n)
$$

and so (4) is

$$
\left(\xi_{\nu}^{(1)}, \cdots, \xi_{\nu}^{(n)}\right)^{t}=-A\left(\gamma_{1 v}, \cdots, \gamma_{n \nu}\right)^{t} .
$$

For $q_{\nu}>0$ let $\delta_{i \nu}=q_{\nu}^{\frac{1}{n}} \gamma_{i \nu}$. Then we wish to count $\lambda_{B}\left(\Omega^{+}\right)$, the number of $\xi_{\nu} \in \Omega^{+}$such that

$$
\begin{gathered}
0<\delta_{1 \nu}, \cdots, \hat{o}_{n \nu}<C \\
1 \leq q_{\nu} \leq B
\end{gathered}
$$

(the corresponding $\lambda_{B}\left(\Omega^{-}\right)$will be shown to be bounded). For $q_{\nu} \neq 0$ set

$$
\kappa_{\nu}^{\prime}=\alpha_{0}+\frac{p_{1 \nu}}{q_{\nu}} \alpha_{1}+\cdots+\frac{p_{n \nu}}{q_{\nu}} \alpha_{n}
$$

so that

$$
\begin{gathered}
\xi_{\nu}=q_{\nu} \kappa_{\nu}{ }^{\prime} \\
\kappa_{\nu}{ }^{\prime}=1+\varepsilon_{\nu} / q_{\nu}
\end{gathered}
$$

where

$$
\varepsilon_{\nu}=\alpha_{1} \gamma_{1 \nu}+\cdots+\alpha_{n} \gamma_{n \nu}
$$

For $q_{\nu}>0$

$$
\delta_{i \nu}=\left(\xi_{0} \kappa_{\nu}{ }^{\prime-1}\right)^{\frac{1}{n}} \zeta^{\frac{\nu}{n}} \gamma_{i \nu}
$$

Set

$$
\begin{gathered}
\eta_{i \nu}=\zeta^{\frac{\nu}{n}} \gamma_{i \nu} \\
\kappa_{\nu}=\left(\xi_{0} \kappa_{\nu}{ }^{\prime-1}\right)^{\frac{1}{n}}
\end{gathered}
$$

so that

$$
\hat{o}_{i \nu}=\kappa_{\nu} \eta_{i \nu} .
$$


Lemma 3. Let $\lambda_{B}^{\prime}\left(\Omega^{+}\right)$be the number of solutions, $v$, of

i) $\xi_{\nu}$ is sufficiently large

ii) $0<\max \left(\eta_{1 v}, \cdots, \eta_{n_{\nu}}\right)<10 \xi_{0}^{-\frac{1}{n}} C=C_{2}$

iii) $0<\eta_{1 \nu}, \cdots, \eta_{n_{\nu}}<C_{\kappa_{\nu}^{-1}}$

iv) $1 \leq \xi_{\nu} \leq 2 B$.

Then $\lambda_{B}\left(\Omega^{+}\right)+0(1) \leq \lambda_{B}^{\prime}\left(\Omega^{+}\right) \leq \lambda_{4 B}\left(\Omega^{+}\right)+0(1)$.

Proof. By i) and ii) we see $q_{\nu} \neq 0$ since $q_{\nu}=0$ implies for $\eta_{i \nu}>0$

$$
C_{2}>\eta_{i \nu}=\zeta^{\frac{\nu}{n}}\left(-p_{i \nu}\right) \geq \zeta^{\frac{\nu}{n}}=\xi_{0}^{-\frac{1}{n}} \frac{1}{\xi_{\nu}}
$$

which violates i). Now by i) $\zeta^{\nu}$ is large so by ii) and (13) $\gamma_{i \nu}$ is small so by (11) $\varepsilon_{\nu}$ is small and so from (10) we may assume $\frac{1}{2} \leq \kappa_{\nu}{ }^{\prime} \leq \frac{3}{2}$. From this and (9) we have $q_{\nu}$ is large and positive. In this situation (7) and iii) are equivalent (see. (15)). Finally by iv) $q_{\nu}=\xi_{\nu} \kappa_{\nu}{ }^{-1} \leq 4 B$. Thus the right hand inequality is true. Conversely assume (7) and (8). We may assume $q_{\nu}$ is large. So from (7), (10), (11), (12) we see that $\kappa_{\nu}{ }^{\prime}$ is close to 1 and so by (9) $\xi_{\nu}$ is large. Again (7) and iii) are equivalent. Here iii) implies ii). Finally by (8), $\xi_{\nu}=q_{\nu} \kappa_{\nu}{ }^{\prime} \leq 2 B$ and so we are done.

It follows from the above argument that solutions of $*$ are such that $\xi_{v}$ and $q_{v}$ have the same mangitude; in particular $\xi_{\nu}>0$ and so $\lambda_{B}\left(\Omega^{-}\right)=0(1)$.

So now we know it suffices to show that if there are an infinite number of solutions, $\lambda_{B}^{\prime}\left(\Omega^{+}\right) \sim C_{1} \log B$ (with $C_{1}>0$ ).

\section{Counting the relevant units}

We now essentially prove the theorem except that instead of counting $1 \leq q_{\nu} \leq B$ we count $1 \leq \nu_{1} \leq N$. We then put $B$ back.

For $1 \leq i \leq r+s$ set

$$
X_{i}=\left(\log \left|\zeta_{i}^{\frac{1}{n}} \zeta_{i}^{(2)}\right|, \cdots, \log \left|\frac{1}{\zeta_{i}^{n}} \zeta_{i}^{(r+s)}\right|, \arg \zeta_{i}^{(r+1)}, \cdots, \arg \zeta_{i}^{(r+s)}\right)^{t}
$$

where we assume for $Z \in C$ that the argument of $Z$ satisfies $0 \leq \arg Z<2 \pi$. So $X_{i} \in \boldsymbol{R}^{n-1}$. Further for $r+s+1 \leq i \leq n$ set $X_{i}=(0, \cdots, 0,2 \pi, 0, \cdots, 0)^{t}$ with $2 \pi$ in the $i^{\text {th }}$ spot.

Now $\operatorname{det}\left(X_{1}, \cdots, \hat{X}_{i}, \cdots, X_{n}\right)=0$ for $i=1,2, \cdots, r+s$ violates the known rank for the regulator matrix. So without loss of generality (rela- 
beling $\left.\zeta_{1}, \cdots \zeta_{r+s}\right)$ we may assume

$$
\operatorname{det}\left(X_{2}, \cdots, X_{n}\right) \neq 0 \text {. }
$$

Lemma 4. Let $\#_{N}^{\prime}$ be the number of solutions of the inequalities

$$
\begin{gathered}
0<\eta_{1 \nu}, \cdots, \eta_{n_{\nu}}<C_{3} \\
1 \leq \nu_{1} \leq N \quad\left(\text { or } 1 \leq-\nu_{1} \leq N\right) .
\end{gathered}
$$

Then

$$
H_{N}^{\prime} \sim C_{4} N \quad(N \rightarrow \infty)
$$

where $C_{4}>0$ is some constant.

Proof. Set

$$
A_{1}=-\left(\begin{array}{llll}
\xi_{0}^{(1)-1} & & & 0 \\
& \cdot & & \\
& & \cdot \xi_{0}^{(n)-1}
\end{array}\right) A
$$

with $A$ as in (3). Also set $\rho_{i \nu}=\zeta^{\frac{\nu}{2}} \zeta^{(i)_{\nu}}(1 \leq i \leq n)$. Then by (6) and (13)

$$
A_{1}\left(\eta_{1 v}, \cdots, \eta_{n \nu}\right)^{t}=\left(\rho_{1 \nu}, \cdots, \rho_{n \nu}\right)^{t} \text {. }
$$

Now $\rho_{r+s+\imath, \nu}=\bar{\rho}_{r+i, \nu}(1 \leq i \leq s)$ so we omit the last $s$ coordinates in the vector of $\rho$ 's.

That is, define the linear transformation $A_{2}$ by

$$
\begin{aligned}
& \boldsymbol{R}^{n} \stackrel{A_{1}}{\longrightarrow} \boldsymbol{R}^{r} \times \boldsymbol{C}^{2 s} \stackrel{\text { proj. }}{\longrightarrow} \boldsymbol{R}^{r} \times \boldsymbol{C}^{s}=\boldsymbol{R}^{n} \text { so } \\
& A_{2}\left(\eta_{1 \nu}, \cdots, \eta_{n_{\nu}}\right)^{t}=\left(\rho_{1 \nu}, \cdots, \rho_{r+s, \nu}\right)^{t} .
\end{aligned}
$$

We show $A_{2}$ is non singular. We know from (3) of Lemma 1 that $A$ is non singular and so also $A_{1}$ is non sigular (i.e., $\operatorname{det} A_{1} \neq 0$ ). Now if the row vectors of $A_{1}$ are $A^{(1)}, \cdots, A^{(n)}$ we see $A^{(r+i)}=\bar{A}^{(r+s+i)}(1 \leq i \leq s)$ and that the row vectors of $A_{2}$ are $A^{(1)}, \cdots, A^{(r)}, \operatorname{Re}\left(A^{(r+1)}\right), \cdots, \operatorname{Re}\left(A^{(r+s)}\right)$, $\operatorname{Im}\left(A^{(r+1)}\right), \cdots, \operatorname{Im}\left(A^{(r+s)}\right)$. So we see that $\operatorname{det} A_{2}=2^{-s} \operatorname{det} A_{1} \neq 0$, as $\operatorname{desired}$.

Now

$$
\begin{aligned}
\rho_{1 \nu} \rho_{2 \nu} \cdots \rho_{n \nu} & =\rho_{1 \nu} \cdots \rho_{r \nu}\left|\rho_{r+1, \nu}\right|^{2} \cdots\left|\rho_{r+s, \nu}\right|^{2} \\
& =\left(N \zeta_{1}\right)^{\nu_{1}} \cdots\left(N \zeta_{r+s}\right)^{\nu_{r+s}} \\
& = \pm 1
\end{aligned}
$$

So the images of the points $\left(\eta_{1 v}, \cdots, \eta_{n_{\nu}}\right)$ under $A_{2}$ lie on the surface $\mathscr{S}$ : 


$$
\begin{gathered}
u_{1} u_{2} \cdots u_{r}\left|u_{r+1}\right|^{2} \cdots\left|u_{r+s}\right|^{2}= \pm 1 \text { in } \boldsymbol{R}^{n}=\boldsymbol{R}^{r} \times \boldsymbol{C}^{s}\left(\text { so }\left(u_{1}, \cdots, u_{r}\right) \in \boldsymbol{R}^{r},\right. \\
\left.\left(u_{r+1}, \cdots, u_{r+s}\right) \in \boldsymbol{C}^{s}\right) \text {. Define } \phi: \mathscr{S} \rightarrow \boldsymbol{R}^{n-1} \text { by } \\
\phi\left(u_{1}, \cdots, u_{r+s}\right)=\left(\log \left|u_{2}\right|, \cdots, \log \left|u_{r+s}\right|, \arg u_{r+1}, \cdots, \arg u_{r+s}\right)^{t} \text {. }
\end{gathered}
$$

Then on any part of $\mathscr{S}$ where the signs of $u_{1}, \cdots, u_{r}$ are fixed we see $\phi$ is 1-1 and has as image

$$
\mathscr{D}=\left\{\left(x_{1}, \cdots, x_{n-1}\right) \mid 0 \leq x_{i}<2 \pi \text { for } r+s \leq i<n\right\} .
$$

Now our problem is to count the number of $\left(\eta_{1 v}, \cdots, \eta_{n_{v}}\right)$ lying in an open box in $\boldsymbol{R}^{n}$. This then is equivalent to counting the number of $\left(\rho_{1 \nu}, \cdots, \rho_{r+s, v}\right)$ lying in some open parallelepiped in $\boldsymbol{R}^{n}=\boldsymbol{R}^{r} \times \boldsymbol{C}^{s}$ which meets $\mathscr{S}$ in a set open in $\mathscr{S}$. Then again restricting ourselves to a portion of $\mathscr{S}$ with the signs of $u_{1}, \cdots, u_{r}$ fixed we see the image $\mathscr{R}$ under $\phi$ of this subset of $\mathscr{S}$ is a bounded open set together with some of its boundary. Moreover it is clear that the volume of the boundaries of all the sets involved is zero. We wish to count the number of $\phi\left(\rho_{1 \nu}, \cdots, \rho_{r+s, \nu}\right)$ lying in $\mathscr{R}$.

We see readily

$$
\phi\left(\rho_{1 \nu}, \cdots, \rho_{r+s, \nu}\right) \equiv \nu_{1} X_{1}+\cdots+\nu_{r+s} X_{r+s}(\bmod 2 \pi)
$$

where the congruence is read only in the last $s$ coordinates. So when the last $s$ coordinates are reduced $\bmod 2 \pi$ we want to count which $\nu_{1} X_{1}+\cdots+$ $\nu_{r+s} X_{r+s}$ lies in $\mathscr{R}$. This is clearly the same as counting the number of $\nu_{1} X_{1}+\cdots+\nu_{n} X_{n} \in \mathscr{R}$ (see the definition of $X_{r+s+1}, \cdots, X_{n}$ ).

Let $\Lambda$ be the lattice in $\boldsymbol{R}^{n-1}$ spanned by $X_{2}, \cdots, X_{n}$ (a lattice by (16)). Thus we now see that we wish to count the number of $\nu_{1} X_{1}\left(1 \leq \nu_{1} \leq N\right)$ lying in $\mathscr{R} \bmod \Lambda$. It is a well known theorem in uniform distribution theory that if $\mathscr{R}$ is a set whose boundary has zero volume, then there is a $C_{4} \geq 0$ such that the number of $\nu_{1} X_{1}$ in $\mathscr{R} \bmod \Lambda, 1 \leq \nu_{1} \leq N\left(1 \leq-\nu_{1} \leq N\right)$ is asymptotic to $C_{4} N$.

It should be recalled that the region $\mathscr{R}$ depended on the sign of the coordinates $\rho_{1 \nu}, \cdots, \rho_{r \nu}$. The sign of $\rho_{i \nu}$ depends only on the sign of $\zeta_{1}^{(i) \nu_{1}}, \cdots, \zeta_{r+s}^{(i) \nu_{r+s}}(1 \leq i \leq r)$ and so for a fixed parity of $\nu_{1}, \cdots, \nu_{r+s}$ the region $\mathscr{R}$ does not change. Then the argument should be repeated as above for these $2^{r+s}$ cases.

This concludes the proof of Lemma 4.

We now solve for $\xi_{\nu}$ in terms of $\nu_{1}$. 
Lemma 5. There is a constant $C_{7} \neq 0$ such that for all $\nu$ satisfying i), ii), iii) of Lemma 3 we have

$$
\log \xi_{\nu}=\nu_{1} C_{7}+0(1) \quad\left(\nu_{1} \rightarrow \pm \infty\right) .
$$

Proof. As in the proof of Lemma 3 we see i) and ii) imply $\kappa_{\nu}{ }^{\prime}$ is close to 1 and so from (10) and (14)

$$
C \kappa_{\nu}^{-1}=C \xi_{0}^{-\frac{1}{n}}\left(1+\varepsilon_{\nu} / q_{\nu}\right)^{\frac{1}{n}}=C_{6}+O\left(q_{\nu}^{-1}\right) .
$$

So we are interested in the $\nu$ such that

$$
0<\eta_{1 \nu}, \cdots, \eta_{n \nu}<C_{6}+0\left(q_{\nu}^{-1}\right) .
$$

The $C_{7}$ will depend only on $\zeta_{1}, \cdots, \zeta_{r+s}$ so it suffices to show (18) for all $\nu$ such that

$$
0<\eta_{1 \nu}, \cdots, \eta_{n \nu}<C_{8} .
$$

Then as in Lemma 4 there is a bounded subset $\mathscr{R} \subseteq \boldsymbol{R}^{n-1}$ such that we want all $\nu_{1} X_{1}+\cdots+\nu_{n} X_{n} \in \mathscr{R}$. This says that for $2 \leq i \leq r+s$

$$
\nu_{2} \log \left|\zeta_{2}^{\frac{1}{n}} \zeta_{2}^{(i)}\right|+\cdots+\nu_{r+s} \log \left|\zeta_{r+s}^{\frac{1}{n}} \zeta_{r+s}^{(i)}\right|=-\nu_{1} \log \left|\zeta_{1}^{\frac{1}{n}} \zeta_{1}^{(i)}\right|+0(1)
$$

Set $X_{i}^{\prime}=\left(\log \left|\zeta_{i}^{\frac{1}{n}} \zeta_{i}^{(2)}\right|, \cdots, \log \left|\zeta_{i}^{\frac{1}{n}} \zeta_{i}^{(r+s)}\right|\right),(1 \leq i \leq r+s)$. Then $\operatorname{det}\left(X_{2}, \cdots, X_{n}\right)$ $\neq 0$, (16), implies $\operatorname{det}\left(X_{2}^{\prime}, \cdots, X_{r+s}^{\prime}\right) \neq 0$, Solving for $\nu_{j}(2 \leq j \leq r+s)$ yields with $Y=-\nu_{1} X_{1}^{\prime}+0(1)$

$$
\begin{aligned}
\nu_{j} & =\frac{\operatorname{det}\left(X_{2}^{\prime}, \cdots, X_{j-1}^{\prime}, Y, X_{j+1}^{\prime}, \cdots, X_{r+s}^{\prime}\right)}{\operatorname{det}\left(X_{2}^{\prime}, \cdots, X_{r+s}^{\prime}\right)} \\
& =(-1)^{j-1} \nu_{1} \frac{\operatorname{det}\left(X_{1}^{\prime}, \cdots, \hat{X}_{j}^{\prime}, \cdots, X_{r+s}^{\prime}\right)}{\operatorname{det}\left(X_{2}^{\prime}, \cdots, X_{r+s}^{\prime}\right)}+0(1) .
\end{aligned}
$$

Thus

$$
\begin{aligned}
\log \xi_{\nu} & =\log \xi_{0}+\nu_{1} \log \zeta_{1}+\cdots+\nu_{r+s} \log \zeta_{r+s} \\
& =\nu_{1} \sum_{j=1}^{r+s}(-1)^{j-1} \frac{\operatorname{det}\left(X_{1}^{\prime}, \cdots, \hat{X}_{j}^{\prime}, \cdots, X_{r+s}^{\prime}\right)}{\operatorname{det}\left(X_{2}^{\prime}, \cdots, X_{r+s}^{\prime}\right)} \log \zeta_{j}+0(1) \\
& =\nu_{1} C_{7}+0(1) .
\end{aligned}
$$

Now $C_{7}=0$ implies

$$
\sum_{j=1}^{r+s}(-1)^{j-1} \operatorname{det}\left(X_{1}^{\prime}, \cdots, \hat{X}_{j}^{\prime}, \cdots, X_{r+s}^{\prime}\right) \log \zeta_{j}=0
$$


and this is the regulator of $\mathcal{O}$. So $C_{7} \neq 0$.

Lemma 6. Let $\#_{N}$ be the number of solutions of i), ii), iii) of Lemma 3 such that $1 \leq \nu_{1} \leq N\left(1 \leq-\nu_{1} \leq N\right)$. Then for some $C_{9}>0$, $\mathbb{H}_{N} \sim C_{9} N$.

Proof. We just observed that we must count the number of $\nu$ such that $\xi_{\nu}$ and $q_{\nu}$ are large, (19) holds and $1 \leq \nu_{1} \leq N\left(1 \leq-\nu_{1} \leq N\right)$. Then examining the proof of Lemma 4 we see that we wish to count the number of $\nu_{1}, 1 \leq \nu_{1} \leq N\left(1 \leq-\nu_{1} \leq N\right)$ such that

$$
\nu_{1} X_{1}+\cdots+\nu_{n} X_{n} \in \mathscr{R}+0\left(q_{\nu}^{-1}\right) \text {. }
$$

Let $F_{N}(\varepsilon)$ be the number lying in $\mathscr{R}_{\varepsilon}$ (all $X \in \boldsymbol{R}^{n-1}$ no further from $\mathscr{R}$ than $\varepsilon)$. Then $F_{N}(\varepsilon) \sim C(\varepsilon) N(N \rightarrow \infty)$. Moreover from the uniform distribution theroy $\lim C(\varepsilon)=C(0)(\varepsilon \rightarrow 0)$. Then given $\varepsilon$ there is an $N_{0}(\varepsilon)$ such that $\left|\nu_{1}\right|>N_{0}(\varepsilon)$ implies $\mathscr{R}+0\left(q_{\nu}^{-1}\right) \subseteq \mathscr{R}_{\varepsilon}$. Now clearly the $q_{\nu}$ yielding solutions to ${ }^{*}$ are such that $q_{\nu} \rightarrow \infty$ so also $\xi_{\nu} \rightarrow \infty$ and so by Lemma $5, \nu_{1} \rightarrow \infty$. Hence there is a constant $C_{10}$ independent of $N$ and $\varepsilon$ such that

$$
\#_{N} \leq F_{N}(\varepsilon)+C_{10} N_{0}(\varepsilon) \text {. }
$$

Thus as $N \rightarrow \infty$

$$
\lim \sup \tilde{H}_{N} / N \leq \lim \sup \left(F_{N}(\varepsilon)+C_{10} N_{0}(\varepsilon)\right) / N=C(\varepsilon) .
$$

Since this is true for all $\varepsilon>0$ we see

$$
\lim \sup { }_{N} / N \leq C(0) \text {. }
$$

Similarly for $\lim$ inf and so $\lim \sharp_{N} / N=C(0)$.

We now prove the theorem. It follows from Lemma 5 that $\xi_{\nu}$ is large if and only if $\nu_{1}$ is large and has the same sign as $C_{7}$; we now restrict $\nu_{1}$ to these values. Set $N=\left|C_{7}\right|^{-1} \log B$. Then $1 \leq \xi_{\nu} \leq 2 B$ implies by Lemma 5

$$
0 \leq \log \xi_{\nu}=\nu_{1} C_{7}+0(1) \leq \log B+0(1)
$$

or

$$
0(1) \leq\left|\nu_{1}\right| \leq N+0(1)
$$

Thus

$$
\lambda_{B}^{\prime}\left(\Omega^{+}\right) \leq \Psi_{N+0(1)}
$$

The lower bound is similar and so there are constants $C_{11}$ and $C_{12}$ such that 


$$
\frac{\#_{N-C_{11}}}{N} \leq \frac{\lambda_{B}^{\prime}\left(\Omega^{+}\right)}{\left|C_{7}\right|^{-1} \log B} \leq \frac{\#_{N+C_{12}}}{N}
$$

and so letting $N$ (hence $B$ ) tend to $\infty$ we have the desired result.

\section{RefERENCES}

[1] W.W. Adams. Simultaneous Asymptotic Diophantine Approximations. Mathematika 14 (1967) 173-180.

[2] W.W. Adams. Simultaneous Asymptotic Diophantine Approximations to a Basis of a Real Cubic Number Field. J. of Number Theory 1 (1969) 179-194.

[ 3 ] S. Lang. Asymptotic Approximation to Quadratic Irrationals. Am. J. of Mathematics 87 (1965) 481-487.

[ 4 ] L.G. Peck. Simultaneous Rational Approximations to Algebraic Numbers. Bull. AMS 67 (1961) 197-201.

[5] W.M. Schmidt. Simultaneous Approximations to a Basis of a Real Algebraic Number Field. Am. J. of Mathematics 88 (1966) 517-527.

Department of Mathematics

University of Maryland 